\title{
Selective Bladder Preservation Using Chemo-Radiotherapy in Treatment of Muscle Invasive Bladder Cancer
}

\author{
HEND M.H.R. ELKALLA, M.Sc.*; SOUMAYA M.A. ETEBA, M.D.*; HASSAN ABOL-ENEIN, M.D.**; \\ AHMED H.A. EL-SHAHAT, M.D.* and MONA M.A. FODA, M.D.* \\ The Departments of Clinical Oncology \& Nuclear Medicine* and Urology \& Nephrology Center**, Faculty of Medicine, \\ Mansoura University
}

\begin{abstract}
Background: This study evaluated the outcomes of patients with muscle-invasive bladder cancer (MIBC) stage T2-4a managed by tri-modality therapy by trans-urethral resection (TURB) and concomitant chemo-radiotherapy (CRT).

Aim of Study: We were aiming for preservation of bladder.

Patients and Methods: This study was a prospective randomized clinical trial including 43 patients with MIBC (T2-T4a N0) presented to Clinical Oncology Department, Mansoura University Hospital \& Urology center during the period from $5 / 2008$ to $9 / 2010$. Patients were randomized to 2 arms: (I): Included 24 patients, who underwent TURB then concomitant CRT using cisplatin plus paclitaxel, while (II): Included 19 patients received same protocol with cisplatin and 5. Flurouracil. All patients who showed complete response (CR) after induction and consolidation phases were given adjuvant chemotherapy 4 cycles every 3 weeks.
\end{abstract}

Results: In arm I: $65.2 \%$ completed the treatment protocol. In arm II: $63.2 \%$ completed the treatment protocol. In arm I: $82.6 \%$ showed complete response (CR), $4.3 \%$ showed disease progression (DP) \& $13 \%$ had only partial response (PR). Patients who achieved CR entered the consolidation phase of treatment. In arm II, 78.9\% showed CR, 21.1\% showed PR. The PFS was $78.26 \%$ \& $68.42 \%$ for both arms respectively. The 3 -year OS was slightly better for arm II. The 3-year OS was $60.87 \% \& 68.42 \%$ for both arms respectively.

Conclusions: Bladder preservation is a good choice for treatment of MIBC but with good selection of the cases and careful follow up to avoid major toxicities which lead to interruption or stoppage of the preservation protocol.

Key Words: Bladder cancer - Tri-modality treatment - Chemoradiotherapy - Bladder preservation.

\section{Introduction}

BLADDER cancer (BC) is the 4 th most common malignancy among men in the Western world \&

Correspondence to: Dr. Hend M.H.R. Elkalla, E-Mail: dr.hend1113@gmail.com accounts for approximately $5-10 \%$ of all cancers in Europe and U.S [1]. Bladder cancer (BC) is the ninth most common cancer worldwide. More than $60 \%$ of all $\mathrm{BC}$ cases and half of deaths occur in less developed regions of the world [2]. Men to women ratio of bladder cancer is 3:1 approximately [3]. According to the results of the National Population-Based Registry Program of Egypt 20082011 ; BC was the 2 nd most common cancer in males \& the 3 rd in both sexes $(10.71 \& 6.94 \%$ respectively) [4]

In general, Muscle-invasive bladder cancer (MIBC) constitutes about $30 \%$ of newly diagnosed cases, with about $70 \%$ being non-invasive (Ta, Tis, T1). About $20-30 \%$ of non-invasive BC cases progress to invasive cancer after transurethral resection of bladder tumor (TURB) [5]

Since the 1970s, radical cystectomy with bilateral pelvic lymph node dissection (PLND) has been the standard treatment of muscle-invasive bladder cancer and this supported by many organizations and guidelines such as National Comprehensive Cancer Network (NCCN) and European Association of Urology (EUA) guidelines [6]. Based on data from multicentric randomized controlled trials (RCTs); the role of neoadjuvant chemotherapy before cystectomy is supported for T2,3,4a lesions with negative lymph node (LN) involvement. Neoadjuvant cisplatin-based chemotherapy increases the median survival and lower the recurrence rate without increased treatment related morbidity or mortality [7].

Meta-analysis of RCT reported an overall survival (OS) and disease-specific survival (DSS) benefit in patients with MIBC receiving adjuvant cisplatin-based chemotherapy after RC. The disease 
free survival (DFS) benefit was more obvious in patients with node positive. However the evidence of its use was not strong. These meta-analysis recommended further RCT with adequate sample size [8] . Obviously, bladder preservation is only accepted in the presence of a high cure option with no compromisation of survival expectancy [9] Many investigations focus on optimizing radiation techniques and incorporating more effective systemic chemotherapy and the proper selection of patients based on molecular markers [10].

As the use of combined modality treatment for MIBC has matured, the opportunity for bladder preservation has developed. Preoperative radiation when combined with cisplatin alone, cisplatin and 5 -fluorouracil or cisplatin and paclitaxel results in the down-staging to $\mathrm{T} 0$ of a significant proportion of patients when TURB, radiation and multi-agent chemotherapy are combined, CR rates of $70 \%$ or greater have been achieved. The radiation sensitizing effects of cisplatin have been long recognized, and the opportunity to safely enhance this effect by the simultaneous administration of a second radiation sensitizer such as 5-Fluorouracil or paclitaxel has been a goal of RTOG protocols since 1995 [11].

\section{Patients and Methods}

This study is a prospective randomized clinical trial which included 43 patients with muscle invasive bladder cancer (MIBC); T2- T4a N0 M0 who presented to Clinical Oncology \& Nuclear Medicine Department, Mansoura University Hospital and Urology Center during the period from May 2008 to September 2010.

\section{Selection criteria:}

- Operable patients with muscle invasive bladder cancer, all histological types.

- AJCC Stages T2-4a N0 M0.

- ECOG performance scale 0-1.

- Patients must have a transurethral resection of the bladder tumor (TURB) as judged safely as possible.

- Hemoglobin level (Hgb) $\geq 10 \mathrm{mg} / \mathrm{dl}$; white blood cells count (WBC) > $4000 / \mathrm{ml}$ and Platelet count $\geq 100,000 / \mathrm{mm}^{3}$

- Serum creatinine $<1.5 \mathrm{mg} \%$; creatinine clearance of $60 \mathrm{ml} / \mathrm{min}$ or greater.

- Treatment must begin within 8 weeks following transurethral resection of bladder tumor and endoscopic evaluation.

- Patients must sign study specific informed consent form prior to study entry.

\section{Pre-treatment evaluation:}

- History was taken from all patients. They were examined physically including weight, height and surface area. Performance status was assessed.

- Investigations included:

- Laboratory studies: Complete blood count, liver function tests, serum creatinine, creatinine clearance and serum alkaline phosphatase.

- Radiological evaluation: Chest X-ray, CT or MRI and bone scan.

- Endoscopic evaluation by cystoscopic examination, bimanual examination under anesthesia, multiple punch biopsies and complete transurethral resection as thorough as possible.

- Pathological examination of the biopsy taken by endoscope for pathological type, grade and depth of muscle invasion and urine cytology examination.

- Stage of disease was determined according to AJCC staging (2002).

- Assessment of the performance status was done according to Eastern Co-Operative Oncology Group (ECOG).

\section{Treatment:}

For patients with T2-T4a NO M0 MIBC and who were candidates for radical cystectomy, TURB was done then induction and consolidation CRT were received in the form of paclitaxel, cisplatin, and irradiation (TCI) in Arm I or 5-FU, cisplatin, and irradiation (FCI) in Arm II. The irradiation used was accelerated hyper-fractionation for the tumor with a standard dose schedule for the pelvis.

\section{Surgery:}

TURB as much as is judged safely possible then after induction chemo-radiotherapy endoscopic response evaluation was done in week 7 following the completion of the induction CRT by multiple punch biopsies. If there was response in the form of no malignancy (T0), papillary tumor (Ta) or carcinoma in situ (Tcis), the patient would enter consolidation phase. If there was no response in the form of $\mathrm{T} 1$ or more, the patient was converted to radical cystectomy at week 9 .

\section{Chemo-radiotherapy:}

Induction phase: All patients after TURB entered in induction phase of concomitant CRT within 8 weeks from TURB. Patients were randomized to one of two treatment arms for 3 weeks (week $1->3)$ : 
Arm I: Patients received concomitant CRT in the form of:

- Paclitaxel 50mg/m² (Day 1, 8, 15).

- Cisplatin $15 \mathrm{mg} / \mathrm{m}^{2}$ (Day $1 \rightarrow 3,8 \rightarrow 10,15 \rightarrow 17$ ).

- Accelerated hyperfractionated RT, two sessions per day in the form of $1.6 \mathrm{~Gy}$ to small pelvic fields then $1.5 \mathrm{~Gy}$ boost to whole bladder 4-6 hours apart (day $1 \rightarrow 5,8 \rightarrow 12,15 \rightarrow 17$ ) i.e: bid $\mathrm{x} 13$ days ( 26 fractions with a total dose of 40.3Gy).

Arm II: Patients received concomitant CRT in the form of:

- 5-FU $400 \mathrm{mg} / \mathrm{m}^{2}$ (Day $1 \rightarrow 3,15 \rightarrow 17$ continuous I.V infusion).

- Cisplatin $15 \mathrm{mg} / \mathrm{m}^{2}$ (Day $1 \rightarrow 3,8 \rightarrow 10,15 \rightarrow 17$ ).

The radiotherapy given was the same as in arm I inclusive.

Consolidation phase: All patients who showed response to induction treatment after interassessment entered in a consolidation phase of concomitant CRT at week 8 for 2 weeks. Each patient continued with his original arm for 2 weeks; week 8,9 .

Arm I: Patients received concomitant CRT in the form of:

- Paclitaxel $50 \mathrm{mg} / \mathrm{m}^{2}$ (Day 1, 8).

- Cisplatin $15 \mathrm{mg} / \mathrm{m}^{2}$ (Day $1 \rightarrow 3,8 \rightarrow 10$ ).

- Accelerated hyperfractionated RT, two sessions per day with a dose of $1.5 \mathrm{~Gy}$ to small pelvic fields then $1.5 \mathrm{~Gy}$ again to small pelvic fields 46 hours apart (day $1 \rightarrow 5,8 \rightarrow 10$ ) i.e: bid $\mathrm{x} 8$ days (16 fractions with a total dose of $24 \mathrm{~Gy}$ ).

Arm II: Patients received concomitant CRT in the form of:

- 5-FU $400 \mathrm{mg} / \mathrm{m}^{2}$ (Day $1 \rightarrow 3,8 \rightarrow 10$ continuous I.V infusion).

- Cisplatin $15 \mathrm{mg} / \mathrm{m}^{2}$ (Day $1 \rightarrow 3,8 \rightarrow 10$ ).

The radiotherapy given was the same as in arm I inclusive.

Adjuvant phase: All patients who ended consolidation phase or those who underwent radical cystectomy entered the adjuvant phase after one month from their last line of treatment whatever it was chemo-radiotherapy or radical cystectomy.

Adjuvant phase included 4 cycles of chemotherapy which were given 3 weeks apart. Chemotherapeutic agents were gemcitabine, paclitaxel and cisplatin according to the following protocol:
- Gemcitabine 1000mg/m² (Day 1,8).

- Paclitaxel 50mg/m² (Day 1,8).

- Cisplatin 35mg/m² (Day 1,8).

Radiotherapy technique:

- Radiotherapy given during induction:

Treatment schedule: External beam irradiation, 1.6 Gy, was delivered to the small pelvic field in the first RT session followed by an inter fraction period of at least 4-6 hours. During the second RT session, $1.5 \mathrm{~Gy}$ was delivered to the whole bladder with a safety margin. The bladder was full before the treatment session to the small pelvic fields and was empty before the treatment session for the whole bladder field.

Target volumes:

- Small pelvic fields:

The field included the whole bladder, the gross tumor volume, the prostate and the prostatic urethra, and the lymph nodes immediately adjacent to the bladder. The fields were designed using a simulator with the patient having 40 to $50 \mathrm{ml}$ air contrast cystogram (20-30ml dye $+20 \mathrm{ml}$ air).

The combination of four shaped anterior, posterior, and lateral fields were used. In the cranialcaudal dimension, the planning target volume (PTV) extended from the lower pole of the obturator foramen to the anterior aspect of the S 1-S2 junction. In the anterior and posterior pelvic field, PTV width extended $1.5 \mathrm{~cm}$ lateral to the bony margin of the pelvis at its widest point. The anterior and posterior fields had been shaped with inferior corner blocks, which shielded the medial border of the femoral heads. For the two parallel-opposed lateral fields, the anterior boundary of the PTV was $1.0 \mathrm{~cm}$ anterior to the most anterior portion of the bladder mucosa seen on the air contrast cystogram. Posteriorly, the PTV extended at least $1.5 \mathrm{~cm}$ posterior to the most posterior portion of the bladder or $1.5 \mathrm{~cm}$ posterior to the bladder tumor mass if it was palpable or identifiable on the pelvic CT scan.

- Whole bladder fields:

These fields included the whole bladder plus safety margin $1.5 \mathrm{~cm}$ all around and were designed during the same simulation with the same air contrast cystogram. Three field techniques were used for the bladder boost in the form of one anterior field and two direct lateral wedged fields or two lateral posterior wedged fields.

\section{- Radiotherapy given during consolidation:}

Consolidation therapy started 7-14 days following a cystoscopic re-evaluation demonstrating a 
complete response to the induction therapy. A dose of $1.5 \mathrm{~Gy}$ (per fraction) was given to the small pelvic field in two RT sessions per day, with an interfraction period of at least 4-6 hours.

The previously simulated small pelvic fields were treated during the consolidation phase.

\section{Radiation dose specification:}

The induction radiotherapy course delivered $20.8 \mathrm{~Gy}$ to the small pelvic fields and $40.3 \mathrm{~Gy}$ to the bladder with the tumor and safety margin. The radiation given during the consolidation treatment was $24 \mathrm{~Gy}$ to the pelvis and the whole bladder. Radiotherapy given in both phases resulted in a total dose to the bladder with the tumor volume and safety margin of $64.3 \mathrm{~Gy}$ over 8 weeks in 42 fractions and a total dose of $44.8 \mathrm{~Gy}$ to the pelvic lymph nodes.

\section{Patients follow-up:}

\section{- During treatment:}

Patients were followed up during radiotherapy weekly for acute radiation toxicity according to World Health Organization (WHO) for small bowel and urinary tract toxicity. Early effects were recorded weekly during treatment and after 4 weeks.

Patients were followed up during chemotherapy for symptoms \& sign of toxicity either hematological or non-hematological toxicity using WHO cancer toxicity criteria for grades of toxicity.

\section{- After the end of treatment:}

Patients were followed up every 1-2 month by clinical examination, every 3 months by abdominopelvic CT or MRI and endoscopy after end of treatment then when needed.

Treatment regimens were compared together. Study primary end point was to evaluate response rate after induction phase of treatment. The secondary endpoints were to evaluate the overall survival \& progression free survival for three years, failure rates (local and distant) and treatment toxicities.

\section{Statistical analysis:}

Data entry and analyses were performed using SPSS statistical package version 10 (SPSS, Inc., Chicago, IL, USA). The quantitative data were presented as a mean, standard deviation, median and range. Student $t$-test was conducted to compare the mean of continuous variable for two different groups of individuals. The qualitative data were presented as number and percentage. The chi- square $\left(\mathrm{X}^{2}\right)$ was used to find the association between variables of qualitative data. Kaplan-Meier Survival Analysis was used to find out overall survival and progression free survival. Relative risk and 95\% confidence intervals were calculated for factors affecting response. The $p$-value of $<-0.05$ and $<0.001$ indicate significant and highly significant results respectively at confidence interval $95 \%$.

\section{Results}

Out of the 43 eligible patients who entered the study, one patient died before completion of the induction phase due to unrelated cause and was excluded from the study.

Patient characteristics: Characteristics of the patients and tumors are summarized in the table below (Table 1). Almost, the base line characteristics of the patients were well balanced between the two treatment groups.

Table (1): Patients' characteristics.

\begin{tabular}{|c|c|c|c|c|c|c|}
\hline \multirow{2}{*}{$\begin{array}{l}\text { Patient } \\
\text { characteristic }\end{array}$} & & \multicolumn{2}{|c|}{$\begin{array}{l}\text { Arm I } \\
(n=23)\end{array}$} & \multicolumn{2}{|c|}{$\begin{array}{l}\text { Arm II } \\
(\mathrm{n}=19)\end{array}$} & \multirow{2}{*}{$\begin{array}{c}p- \\
\text { value }\end{array}$} \\
\hline & & No. & $\%$ & No. & $\%$ & \\
\hline Age (Years) & $\begin{array}{l}<60 \\
\geq 60\end{array}$ & $\begin{array}{l}4 \\
19\end{array}$ & $\begin{array}{l}17.4 \\
82.6\end{array}$ & $\begin{array}{l}2 \\
17\end{array}$ & $\begin{array}{l}10.5 \\
89.5\end{array}$ & 0.673 \\
\hline Sex & $\begin{array}{l}\mathrm{M} \\
\mathrm{F}\end{array}$ & $\begin{array}{l}23 \\
-\end{array}$ & $\begin{array}{l}100 \\
-\end{array}$ & $\begin{array}{l}16 \\
3\end{array}$ & $\begin{array}{l}84.2 \\
15.8\end{array}$ & 0.084 \\
\hline Tumor stage & $\begin{array}{l}\text { T2 } \\
\text { T3a } \\
\text { T3b } \\
\text { T4a }\end{array}$ & $\begin{array}{l}9 \\
1 \\
13 \\
-\end{array}$ & $\begin{array}{l}39.1 \\
4.3 \\
56.5 \\
-\end{array}$ & $\begin{array}{l}6 \\
11 \\
2\end{array}$ & $\begin{array}{l}31.6 \\
\\
57.9 \\
10.5\end{array}$ & 0.332 \\
\hline Tumor grade & $\begin{array}{l}\text { G I } \\
\text { G II } \\
\text { G III }\end{array}$ & $\begin{array}{l}1 \\
2 \\
20\end{array}$ & $\begin{array}{l}4.3 \\
8.7 \\
87\end{array}$ & $\begin{array}{l}2 \\
2 \\
15\end{array}$ & $\begin{array}{l}10.5 \\
10.5 \\
78.9\end{array}$ & 0.714 \\
\hline Performance status & $\begin{array}{l}\text { G } 0 \\
\text { G } 1\end{array}$ & $\begin{array}{l}22 \\
1\end{array}$ & $\begin{array}{l}95.7 \\
4.3\end{array}$ & $\begin{array}{l}13 \\
6\end{array}$ & $\begin{array}{l}68.4 \\
31.6\end{array}$ & 0.034 \\
\hline
\end{tabular}

Primary end point: The response to induction phase was evaluated in week 7 after completion of induction chemo-radiotherapy. Inter-assessment was done by cystoscopic examination and multiple punch biopsies. In arm I: One patient (4.3\%) showed disease progression (DP), 3 patients (13\%) had only partial response (PR) and 19 patients (82.6\%) showed complete response (CR). Patients who achieved $\mathrm{CR}$ entered the consolidation phase of treatment protocol. In arm II, 4 patients (21.1\%) showed PR and 15 patients (78.9\%) showed CR, so they entered the consolidation phase as shown in Fig. (1). There was no significant difference between both arms regarding the response to induction phase of treatment protocol $(p=0.537)$. 




Fig. (1): Response to treatment in both arms.

All patients ( 10 patients in arm I \& 6 patients in arm II) with early stage tumor T2+T3a showed CR $(100 \%)$ in both arms of the study. On the other hand, patients with advanced tumors T3b+T4a showed CR in $69.2 \%$ of patients ( 9 patients in arm I \& 9 patients in arm II). There was no statistically significant difference between both groups regarding response $(p=0.1 \& 0.25$ in both arms respectively).

\section{Secondary end points:}

\section{$P F S \&$ OS rates:}

The median duration of follow-up was 16 months (range: 2-35 months) with a mean duration of $15.26 \pm 9.88$ months.

Arm I demonstrated a superior PFS at 3-years compared with arm II. The PFS was $78.26 \%$ \& $68.42 \%$ for both arms respectively. The median PFS was $21 \& 18$ months in both arms respectively. Similarly, PFS at 6 months, 1 -year and 2-years were better in arm I of the study. The corresponding PFS values were $87 \%, 78.26 \%$ and $78.26 \%$ for arm I. On the other hand, PFS values for arm II were $85 \%, 73.68 \%$ and $68.42 \%$ respectively. However there were no statistically significant differences in PFS between both arms $(p=0.485)$ as shown in Fig. (2).

Overall survival at 3-years was slightly better for arm II. The 3-year OS was $60.87 \%$ \& $68.42 \%$ for both arms respectively. The median OS was 25 $\& 23$ months for both arms respectively $(p=0.683)$. There were no statistically significant differences between both arms regarding OS at 3-years. OS at 6 months, 1-year and 2-years were 77\%, $69.57 \%$ and $65.22 \%$ respectively for arm I. On the other hand, the corresponding figures for arm II were $79 \%, 78.95 \%$ and $68.42 \%$ respectively as shown in Fig. ( 3).

Univariate analysis was done to study the impact of various prognostic factors on PFS \& OS and we found that tumor stage is the only factor that affected PFS with statistical significance but other factors were non-significant.

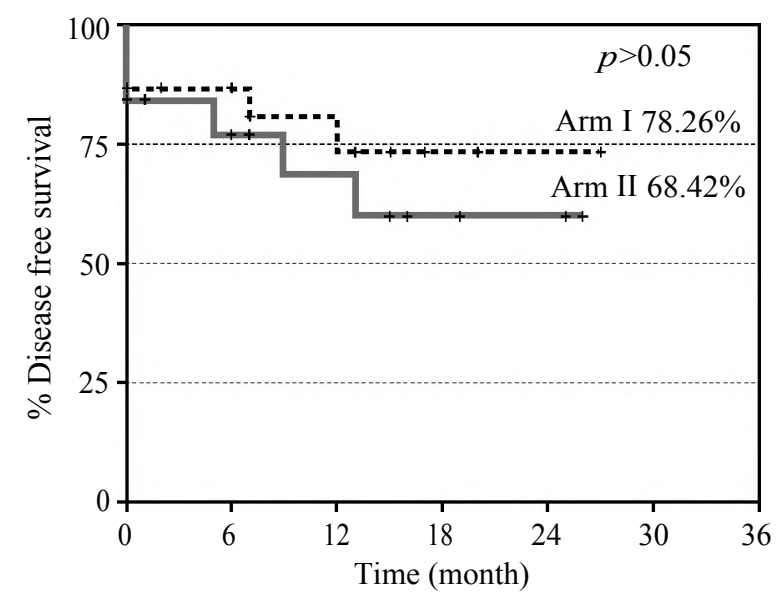

Fig. (2): PFS in both arms.

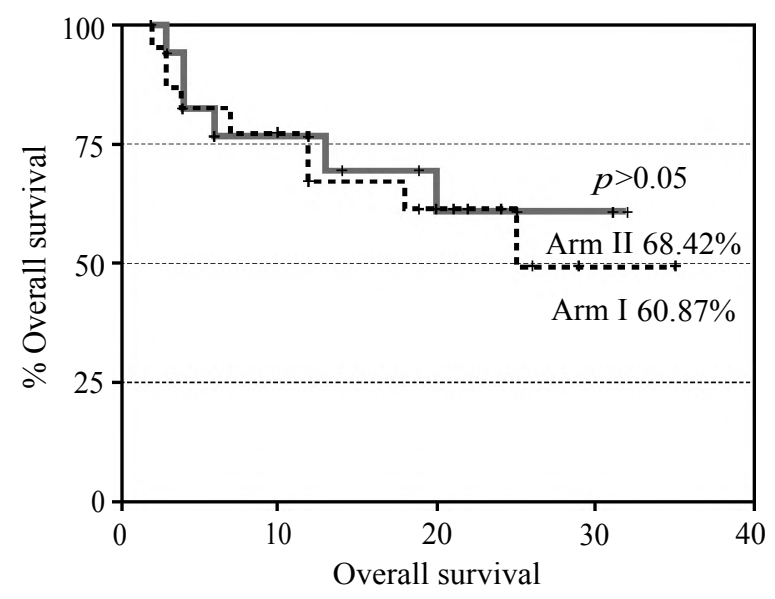

Fig. (3): Os in both arms.

\section{Failure rates:}

Loco-regional failure was found in one out of 23 patients $(4.3 \%)$ in arm I. Local recurrence occurred in the bladder and extended outside the wall to perivesical fat. On the other hand in arm II, 2 out of 19 patients $(10.5 \%)$ developed local recurrence, one case extended to pelvic lymph nodes and the other case developed recurrence at the bladder and extended throughout the wall to perivesical fat and surrounding pelvic organs; the rectum. There were no statistically significant differences between both arms $(p=0.581)$. 
Distant metastasis occurred in 3 out of 23 patients (13\%) in arm I. Disease metastasized to bone in one case and in the second two cases to lung and bone. In arm II, 3 out of 19 patients (15.8\%) had distant metastases. One case developed lung, bone and liver metastases and the other two cases developed only bone metastases. There was no statistically significant difference between both arms regarding occurrence of distant metastasis.

\section{Treatment toxicity:}

All patients were evaluated for toxicities according to WHO grading system. The most frequently occurring adverse effects were dysuria, frequency, diarrhea and anemia. Only one patient in arm I developed grade IV neutropenia that was successfully treated. Otherwise no other major hematologic toxicity was encountered in any of the two arms of the study. Dysuria \& frequency were the most frequently observed non-hematologic toxicities followed by diarrhea. Gastrointestinal complications were more or less equivalent in both arms of the study. Side effects were tolerable and manageable. No treatment-related deaths was encountered in any of the two study groups.

\section{Discussion}

Our patients showed an age range from 48-78 years with a median age of 64 years in arm I and 70 years in arm II, this is consisted with the worldwide reported median age. Khosravi-Shahi and Cabezón-Gutiérrez reported that median age at diagnosis was 65 years, and $70 \%$ of patients with bladder cancer were $>60$ years of age [12] Our study showed male predominance with male to female ratio 5.3:1. This ratio is higher than the reported world-wide ratio which is about 3:1 [12] This may be explained by the small number of the sample to be presented with the world wide ratio.

Arm I showed higher CR than arm II but the difference did not reach the statistical significance. In our study, we used cisplatin and paclitaxel plus RT in arm I but in arm II we used cisplatin and 5.flurouracil also plus RT. Our results are comparable with the studies conducted by Sabaa et al. [13], Khader et al. [14] \& Efstathiou et al. [15]. Sabaa et al. studied 104 patients with MIBC that were treated by complete TURB followed by CRT in the form of gemcitabine and cisplatin and conventional radiotherapy after the maximum resection of their tumors. Complete response was shown by $78.8 \%$ [13] \& this typically is equal to the results of arm II. The slightly higher CR rate observed in arm I may be due to the use of paclitaxel which may be more potent than 5.flurouracil and gemcitabine. Khader and his colleagues studied 14 patients with MIBC. Initial therapy consisted of TURB followed by induction chemotherapy, then irradiation with concurrent platinum-based agents. The bladder and pelvic lymphatics were treated via a four-field box technique to a total dose of $4500 \mathrm{cGy}$ (180cGy daily fractions in 5 consecutive days). Additional therapy with irradiation (up to 6400 cGy) was delivered to the bladder with safety margin to complete responding patients [14]. They achieved $73 \% \mathrm{CR}$ and this is much near to CR obtained in our results. Efstathiou et al., reported the MGH experience with selective bladder preservation in the treatment of 348 patients with MIBC (T2-T4a). Patients underwent concurrent cisplatinbased chemotherapy and RT after maximal TURB plus neoadjuvant or adjuvant chemotherapy. Repeat biopsy was performed after 40Gy, with initial tumor response guiding subsequent therapy. Those patients showing CR received boost chemotherapy and RT. CR was achieved in $72 \%$ of patients [15] This agrees with our results as they used multidrug regimen with platinum-based agents concomitant with radiotherapy in addition to using chemotherapy as adjuvant or neoadjuvant in the treatment protocol that may contribute to the higher CR obtained. Other clinical researches using cisplatin in combination with RT in patients with MIBC have demonstrated objective tumor responses reaching up to $80 \%$, with acceptable toxicity. These were observed in the studies conducted by Zouhair et al. [16], Rodel et al. [17], Chen et al. [18] and Hagan et al. [19]. Similarly, other studies also used cisplatin plus radiotherapy after TURB and obtained results comparable to those demonstrated in our study: Weiss and his colleagues had CR that reached up to $90.3 \%$ [20], Perdona et al. showed $88.4 \%$ CR [21] and Joung et al. showed 75\% CR [22]. On the other hand, the results obtained in our series are better than that reported by Gamal El Deen et al. [10], Ibrahem et al. [23] and Nowak-sadzikowska et al. [24] studies. CR was achieved by Gamal El Deen in $67.3 \%$ of 55 patients with MIBC [10]. Our results were much higher in both arms and this may be owing to the use of combination chemotherapy in induction and consolidation phases and also the use of unconventional fractionation in the form of accelerated hyperfractionation. Ibrahem et al., found that $60 \%$ of their patients achieved CR [23]. Similarly, this study showed lower results than ours which may also be attributed to the use of multidrug regimens and unconventional fractionation schedules of radiotherapy in both arms of our study. 
Statistical analysis to study the impact of different prognostic factors on response revealed that tumor stage and tumor grade were the most predictive factors for this initial response.

Ibrahem et al., reported that local failure was recorded in $40 \%$ of patients and distant metastasis was reported in $25 \%$ [23]. Kaufman et al., reported loco-regional failure rate as $27.5 \%$ and distant failure rate of $31.25 \%$ [9]. Sabaa et al. found that local failure rate in their study was $16.2 \%$ out of the evaluable 74 patients and distant failure rate was $24.3 \%$ [13] . Zapatero et al., reported the pattern of failure for all patients and according to the treatment protocol. $24.5 \%$ experienced local bladder relapse and 15\% developed distant metastasis [25] However, this failure rates are more or less far from our results. In our current study, after a median follow-up of 16 months the local failure rate for the whole studied patients was 3 out of 42 patients $(7.1 \%)$. One out of 23 patients $(4.3 \%)$ in arm I and 2 out of 19 patients (10.5\%) in arm II developed local failure. This may be explained by the shorter follow-up duration. On the other hand, distant failure rate for the whole group was 6 out of 42 patients (14.3\%). Three out of 23 patients (13\%) in arm I and 3 out of 19 patients $(15.8 \%)$ in arm II developed distant failure and this much more comparable with Zapatero et al., results [25]. The relative difference between treatment failure rates of our study and other series could be explained by the relatively shorter follow-up period in our study compared to other series that had larger sample size and longer follow-up periods.

Gamal El deen et al., reported treatment toxicity observed among 37 patients who ended chemotherapy and radiotherapy protocol [10]. They are different from our results regarding genitourinary (GU) and gastrointestinal (GI) complications. This may be explained by the use of accelerated hyperfractionation in our series, as it is well known that this altered fractionation increases acute side effects to the irradiated area. Hematological complications more or less are equivalent to our results. Hematological complications were recorded in $27 \%$ of their cases, GI complications in $24.3 \%$ of their cases and GU complications in $10.8 \%$ of their cases. Nowak-Sadzikowska et al., treated 27 patients with CRT. Anaemia mainly of grade I (GI) occurred in $11 \%$, leucopenia of GI in $37 \%$ and thrombocytopenia mainly of G III in $22 \%$. GI and GU complications were mainly of GI and GII. Gastrointestinal complications were occurred in $44 \%$ of patients with G I and G II in $15 \%$ of patients
[24]. These results are much more comparable with our results.

At a median follow-up period of 16 months (range: 2-35 months) with a mean duration of $15.26 \pm 9.88$ months, our protocol reported higher PFS at 3-years in arm I than arm II. They were $78.26 \% \& 68.42 \%$ for both arms respectively. On the contrary, OS at 3-years was slightly better for arm II. The 3-year OS was $60.87 \%$ \& $68.42 \%$ for both arms respectively. Both rates PFS and OS did not show statistically significant difference between both arms $(\mathrm{P}=0.485 \& 0.683$ respectively). Gamal El Deen reported that OS at 3-years was 79.23\% [10]. The 3-year OS for the patients without hydronephrosis that are similar to our patient's selection criteria was $59 \%$. This compares favorably with the prior RTOG protocol 89-03 studied by Shipley et al. at [26] and this is also comparable with our results in both arms. Other studies by Zietman et al. at [27] and Kaufman et al. [28] reported 3 -year OS of $83 \%$ with more or less similar radiation fractionation and similar chemotherapy schedules as in arm II of our study. However, our results are a little a bit lower for both arms. This may be due to the smaller number of our sample in comparison to the two previous studies. Houssett and his colleagues reported $63 \%$ OS [29] and this is comparable with the results of both arms of our study. Ibrahem and his colleagues recorded that the 2-year survival and progression free survival (PFS) rates were $67 \%$ and $58 \%$, respectively [23] The OS of this study is mostly similar to our result and DFS is more or less near to our results in arm II but far from the results of arm I. The results obtained by Joung and his colleagues were that DFS and OS rates for all 20 patients were $51.1 \%$ and $38.6 \%$ at $5 \mathrm{yr}$, respectively [22] and this was markedly different from our results. Of the 80 patients studied by Kaufman et al., 44 survived, with an OS rate of $67 \%$ at 36 months and $56 \%$ at 60 months. DFS rate was $73 \%$ at 36 months and $71 \%$ at 60 months [9]. This study design is the most similar to our study and their OS and DFS at 3years are much more similar to our results in both arms.

\section{Conclusions:}

Bladder preservation protocol is a good choice for treatment of bladder cancer but with good selection of the cases and careful follow-up of them to avoid major toxicities that may lead to interruption or even stoppage of the preservation protocol. Many studies should be continued to study more the effect of this protocol on PFS and OS and patient quality of life. 


\section{References}

1- EVANS C., DEBRUYNE F., PAYNE H., et al.: Bladder cancer: Management and future directions. Eur. Urol., Suppl. 6: 365-373, 2007.

2- FERLAY J., SOERJOMATARAM I., DIKSHIT R., et al.: Cancer incidence and mortality worldwide: Sources, methods and major patterns in GLOBOCAN 2012. Int. J. CA, 136 (5), 2015.

3- JACOBS B., LEE C. and MONTIE J.: Bladder cancer in 2010: How far have we come?. CA J. Clin., 60: 244-272, 2010.

4- IBRAHIM A.S., KHALED H.M., MIKHAIL N.N., et al.: Cancer incidence in Egypt: Results of the national population-based cancer registry program. J. of Can Epidem, 2014.

5- BYUN S.J., KIM J.H., OH Y.K. and KIM B.H.: Concurrent chemoradiotherapy improves survival outcome in muscleinvasive bladder cancer. Radiat. Oncol. J., 33 (4): 294, 2015.

6- MONTEIRO L.L. and KASSOUF W.: Radical Cystectomy is the best choice for most patients with muscle-invasive bladder cancer? Opinion: Yes. Int. Braz J. urol., 43 (2): 184-187, 2017.

7- KITAMURA H., TSUKAMOTO T., SHIBATA T., et al.: Randomised phase III study of neoadjuvant chemotherapy with methotrexate, doxorubicin, vinblastine and cisplatin followed by radical cystectomy compared with radical cystectomy alone for muscle-invasive bladder cancer. Japan Clini. Oncol. Group Study J. COG0209. Ann. of Oncol. J., 25 (6): 1192-1198, 2014.

8- National Comprehensive Cancer Network. NCCN Clinical Practice Guidelines in Oncology: bladder cancer. V.5.2017. Accessed at www.nccn.org/professionals/physician_gls/ pdf/bladder.pdf on December 12, 2017.

9- KAUFMAN D., WINTER K., SHIPLEY W., et al.: Muscle-invading bladder cancer, RTOG protocol 99-06: Initial report of a phase I/II trial of selective bladder-conservation employing TURBT, accelerated irradiation sensitized with cisplatin and paclitaxel followed by adjuvant cisplatin and gemcitabine chemotherapy. J. Clin. Oncol., 26: 379385, 2009.

10- GAMAL EL-DEEN H.: Initial results of retrospective study: preoperative transurethral excision plus chemotherapy and radiation therapy and trial of bladder preservation. J. Egy. Nat. Canc. Inst., 19: 133-146, 2007.

11-ZIETMAN A., HENEY N., KAUFMAN D., et al.: Bladder preservation: Optimizing radiotherapy and integrated treatment strategies. BJU Int., 102: 1345-1353, 2008.

12- KHOSRAVI-SHAHI and CABEZÓN-GUTIÉRREZ L.: Selective organ preservation in muscle-invasive bladder cancer: Reviewof the literature. J. Surg. Oncol., 21: 1722, 2012.

13- SABAA M., EL-GAMAL O., ABO-ELENEN M., et al.: Combined modality treatment with bladder preservation for muscle invasive bladder cancer. J. Urol. Oncol. Semin \& Orig. Invest., 28: 14-20, 2010.

14- KHADERA J., FARAHB N. and SALEMA A.: Bladder preservation by concurrent chemoradiation for muscleinvasive bladder cancer: Applicability in low-income countries. Reports of Pract Oncol. \& Radiother, 16: 178183, 2011.

15- EFSTATHIOU J., SPIEGEL D. and SHIPLEY W.: Longterm outcomes of selective bladder preservation by combined-modality therapy for invasive bladder cancer: The MGH experience. Euro. Urol., 61: 705-711, 2012.

16- ZOUHAIR A., OZSAHIN M., SHNEIDER D., et al.: Invasive bladder carcinoma: A pilot study of conservative treatment with accelerated radiotherapy and concomitant cisplatin. Int. J. Cancer, 96: 350-55, 2001.

17- RÖDEL C., GRABENBAUER G.G., KÜHN R., et al.: Combined- modality treatment and selective organ preservation in invasive bladder cancer: Long term results. J. Clin. Oncol., 20: 3061-3071, 2002.

18- CHEN W., LIAW C., CHUANG C., et al.: Concurrent cisplatin, 5-fluorouracil, leucovorin, and radiotherapy for invasive bladder cancer. Int. J. Radiat. Oncol. Biol. Phys., 56: 726-733, 2003.

19- HAGAN M., WINTER K., KAUFMAN D., et al.: RTOG 97-06: Initial report of phase I-II trial of selective bladder conservation using TURB, twice-daily accelerated irradiation sensitized with cisplatin, and adjuwant MCV combination Chemotherapy. Int. J. Radiat. Oncol. Biol. Phys., 57: 665-672, 2003.

20- WEISS C., ENGEHAUSEN D., KRAUSE F., et al.: Radiochemotherapy with cisplatin and 5-fluorouracil after transurethral surgery in patients with bladder cancer. Int. J. Radiat. Oncol. Biol. Phys., 68: 1072-1080, 2007.

21- PERDONA S., AUTORINO R., DAMIANO R., et al.: Bladder-sparing, combined modality approach for muscleinvasive bladder cancer: A multi-institutional, long-term experience. Cancer, 112:75-83, 2008.

22- JOUNG J., HAN K. and KIM T.: Single institutional experience of bladder-preserving trimodality treatment for muscle-invasive bladder cancer. J. Kor Med. Sci., 23: 598-603, 2008.

23- IBRAHIM S., ABD EL-HAFEEZ Z., MOHAMED E., et al.: Transurethral Resection of Bladder Tumor (TUR-BT) then Concomitant Radiation and Cisplatin Followed by Adjuvant Gemcitabine and Cisplatin in Muscle Invasive Transitional Cell Carcinoma (TCC) of the Urinary Bladder. J. Egypt Natl. Canc. Inst., 19: 77-86, 2007.

24- NOWAK-SADZIKOWSKA J., KOWALSKA T., JAKUBOWICZ J., et al.: Conservative treatment in patients with muscle-invasive bladder cancer by transurethral resection, neoadjuvant chemotherapy with gemcitabine and cisplatin, and accelerated radiotherapy with concomitant boost plus concurrent cisplatin-assessment of response and toxicity. Rep. Pract. Oncol. Radiother, 13: 300-308, 2008.

25- ZAPATERO A., MARTIN DE VIDALES C. and ARELLANO R.: Updated results of bladder-sparing trimodality approach for invasive bladder cancer. J. Urol. Oncol. Semin \& Orig Invest, 28: 368-374, 2010.

26- SHIPLEY W., WINTER K., KAUFMAN D., et al.: Phase III trial of neoadjuvant chemotherapy in patients with invasive bladder cancer treated with selective bladder preservation by combined radiation therapy and chemotherapy: Initial results of Radiation Therapy Oncology Group 89-03. J. Clin. Oncol., 16: 3576-3583, 1998. 
27- ZIETMAN A., SHIPLEY W., KAUFMAN D., et al.: A phase I/II trial of transurethral surgery combined with concurrent cisplatin, 5-fluorouracil and twice-a-day radiation followed by selective bladder preservation in operable patients with muscle-invading bladder cancer. J. Urol., 160: 1673-1677, 1998.

28- KAUFMAN D., WINTER K., SHIPLEY W., et al.: The initial results in muscle-invading bladder cancer of RTOG 95-06: Phase I/II trial of transurethral surgery plus radiation therapy with concurrent cisplatin and 5-fluorouracil followed by selective bladder preservation or cystectomy depending on the initial response. Oncologist, 5: 471476,2000

29- HOUSSET M., DUFOUR B. and MAULARD C.: Concomitant 5-fluorouracil-cisplatin and bifractionated split course radiation therapy for invasive bladder cancer. Proc. Am. Soc. Clin. Oncol., 16: 319-320, 1997.

\section{العلاج التحفظى بإستخلدام العلاج الكيماوى والأشعاعى العاتئ

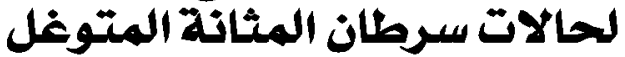

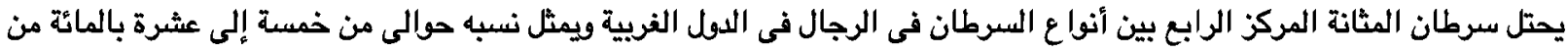

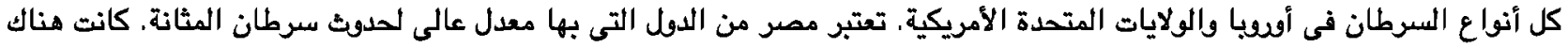

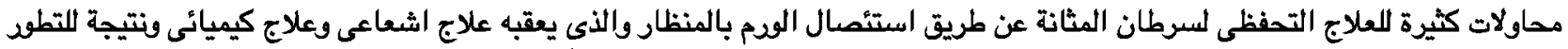

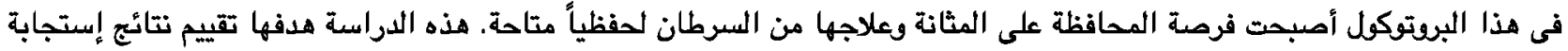

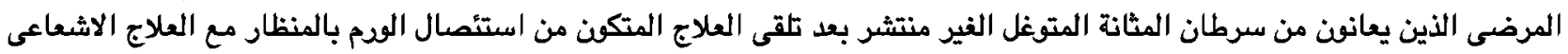

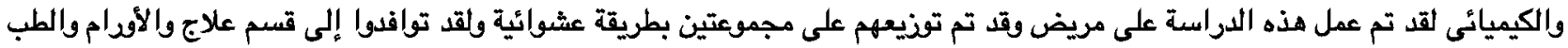

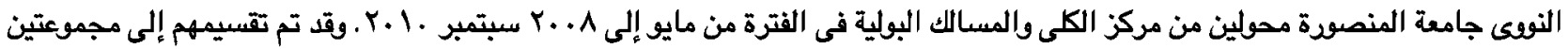

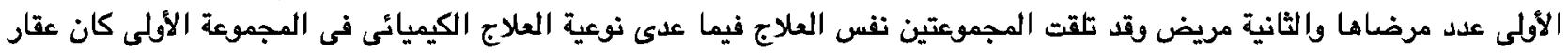

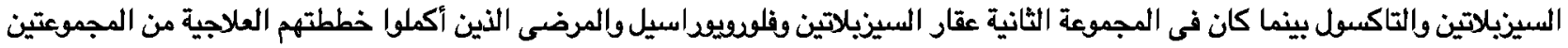

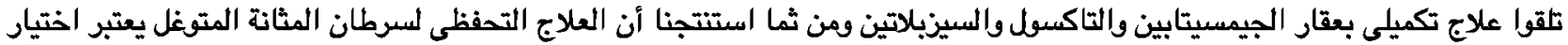

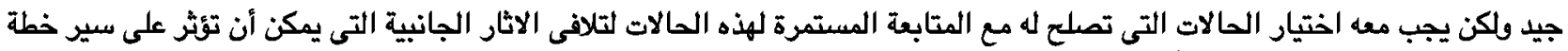
العلاج أو قد تقدى إلى توقفها تماماً نتيجة للذآثار التى لا يمكن تداركها. 